\title{
A new Neptune-mass planet orbiting HD 219828 ^
}

\author{
C. Melo ${ }^{1}$, N. C. Santos ${ }^{2,3,4}$, W. Gieren ${ }^{5}$, G. Pietrzynski ${ }^{5}$, M. T. Ruiz ${ }^{6}$, S. G. Sousa ${ }^{2,7,8}$, F. Bouchy ${ }^{9}$, C. Lovis $^{3}$, \\ M. Mayor ${ }^{3}$, F. Pepe ${ }^{3}$, D. Queloz ${ }^{3}$, R. da Silva ${ }^{3}$, and S. Udry ${ }^{3}$ \\ ${ }^{1}$ European Southern Observatory, Casilla 19001, Santiago 19, Chile \\ e-mail: cmelo@eso.org \\ 2 Centro de Astronomia e Astrofísica da Universidade de Lisboa, Observatório Astronómico de Lisboa, Tapada da Ajuda, \\ 1349-018 Lisboa, Portugal \\ 3 Observatoire de Genève, 51 ch. des Maillettes, 1290 Sauverny, Switzerland \\ 4 Centro de Geofisica de Évora, Rua Romão Ramalho 59, 7002-554 Évora, Portugal \\ 5 Universidad de Concepcion, Departamento de Fisica, Casilla 160-C, Concepcion, Chile \\ 6 Departamento de Astronomia, Universidad de Chile, Casilla Postal 36D, Santiago, Chile \\ 7 Centro de Astrofísica da Universidade do Porto, Rua das Estrelas, 4150-762 Porto, Portugal \\ 8 Departamento de Matemática Aplicada, Faculdade de Ciências da Universidade do Porto, Portugal \\ ${ }^{9}$ Institut d'Astrophysique de Paris, 98bis Bd. Arago, 75014 Paris, France
}

Received 30 November 2006 / Accepted 28 January 2007

ABSTRACT

\begin{abstract}
Two years ago a new benchmark for the planetary survey was set with the discoveries of three extrasolar planets with masses below $20 M_{\oplus}$. In particular, the serendipitous discovery of the $14 M_{\oplus}$ planet around $\mu$ Ara found with HARPS with a semi-amplitude of only $4 \mathrm{~m} \mathrm{~s}^{-1}$ put in evidence the tremendous potential of HARPS for the search of this class of very low-mass planets. Aiming to discovering new worlds similar to $\mu$ Ara b, we carried out an intensive campaign with HARPS to observe a selected sample of northern stars covering a range of metallicity from about solar to twice solar. Two stars in our program were found to present radial velocity variations compatible with the presence of a planet-mass companion. The first of these, HD 219828, was found to be orbited by a planet with a minimum mass of $19.8 M_{\oplus}$ and an orbital period of 3.83 days. It is the 11th Neptune-mass planet found so far orbiting a solar-type star. The radial velocity data clearly show the presence of an additional body to the system, likely of planetary mass. The second planet orbits HD 102195, has a mass of $0.45 M_{\text {Jup }}$ and an orbital period of 4.11 days. This planet has been already announced by Ge et al. (2006, ApJ, 648, 683). Our data confirm and improve the orbital solution found by these authors. We also show that the high residuals of the orbital solution are caused by stellar activity, and use the bisectors of the HARPS cross-correlation function to correct the noise introduced by stellar activity. An improved orbital solution is obtained after this correction. This kind of analysis may be used in the future to correct the radial-velocities for stellar activity induced noise.
\end{abstract}

Key words. stars: individual: HD 219828 - stars: planetary systems - planetary systems: formation - techniques: radial velocities

\section{Introduction}

The serendipitous discovery by Santos et al. (2004a) of a $14 M_{\oplus}$ planet orbiting $\mu$ Ara, simultaneously followed by the announcement of another planet of similar mass around $55 \mathrm{Cnc}$ (McArthur et al. 2004) and of a $21 M_{\oplus}$ mass planet around GJ $436 \mathrm{~b}$ (Butler et al. 2004) set a new benchmark for planet surveys. Since then, a few more cases have been announced (Rivera et al. 2005; Bonfils et al. 2005; Vogt et al. 2005; Udry et al. 2006), including a system of three Neptune-mass bodies (Lovis et al. 2006). For the first time in the literature, the minimum masses of a planet found by Doppler shift techniques were indicated in Earth-masses and not in Jupiter- or Saturn-masses. Even more exciting was the fact that based on their orbital characteristics and masses, it is strongly suggested that these planets may be rocky or icy in nature (Alibert et al. 2006). These discoveries may have thus unveiled the first super-earths, and shown that telluric planets may be common in the solar neighborhood.

The detection of these kinds of planets is not easy to be achieved. For example, the discovery of the $14 M_{\oplus}$ companion

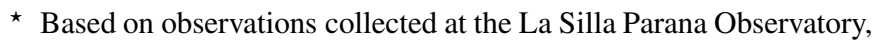
ESO (Chile) with the HARPS spectrograph at the $3.6 \mathrm{~m}$ telescope (Observing runs 075.C-0332, 076.C-0155, and 77.C-0101). of $\mu$ Ara was only made possible thanks to the two main factors. First, the intrinsic stability of an instrument like HARPS which is able to keep a long-term (several years) radial velocity accuracy better than $1 \mathrm{~m} \mathrm{~s}^{-1}$. Second, the observational strategy. $\mu$ Ara was observed in a context of a asteroseismology program, where a unique target was monitored during the whole night and a large number of spectra was collected with exposure times of about a $2 \mathrm{~min}$, in order to measure the stellar acoustic oscillation modes. Seen from the perspective of a radial velocity planetary search, these oscillations are unwanted and are considered as stellar noise. This intrinsic noise is well illustrated by the case of $\mu$ Ara: during the nights when the star was measured with HARPS over a several hours period, the residuals around the orbital fit were merely around $0.4 \mathrm{~m} \mathrm{~s}^{-1}$, a value that increased to near $1.5 \mathrm{~m} \mathrm{~s}^{-1}$ for the remaining nights, when the radial-velocity of the star was an average of only about 15 min integrations (Santos et al. 2004a); this value would be around $3 \mathrm{~m} \mathrm{~s}^{-1}$ if only one single (short exposure) radial-velocity measurement was done. In other words, to be able to achieve the $1 \mathrm{~m} \mathrm{~s}^{-1}$ precision one needs to average the radial velocities for a given star over periods of several minutes (at least 15-20 min), making the searches for very low mass planets very time consuming (see the seismological results on $\mu$ Ara by Bouchy et al. 2005). 
Surprisingly enough, $\mu$ Ara and 55 Cnc were back then the only two stars that were (incidentally) measured with enough precision so that such low mass planets could have been detected. This may imply that very low mass short period planets are very common. This result is supported by recent simulations (Ida \& Lin 2004; Benz et al. 2006) that suggest that very lowmass planets may be more frequent than the previously found giant worlds.

These theoretical results and the discovery of several Neptune-mass planets suggested that if solar type stars are correctly monitored, the number of detection of such low-mass rocky planets can be very high. Having this in mind, we started a project to use the HARPS spectrograph to monitor a sample of about 40 northern stars with metallicity ranging from solar to about twice solar. In the present paper, we present the discovery of a new Neptune-mass planet orbiting the star HD 219828, as well as the confirmation of a Jovian-mass planet orbiting HD 102195. We also find evidence for the existence of a longer period planet companion to the former star. The presentation of the whole sample, along with a detailed study of the behavior of the radial velocities with other properties (e.g. spectral type and activity level) is postponed to a future paper.

\section{Observations}

The observations were carried out using the HARPS spectrograph (3.6-m ESO telescope, La Silla, Chile), in three different observing runs between May 2005 and July 2006 (ESO observing runs 075.C-0332, 076.C-0155, and 77.C-0101). Further measurements of the two stars discussed in this paper were also done in different HARPS GTO runs, in collaboration with the Geneva team.

Radial-velocities were obtained directly using the HARPS pipeline. We refer to Pepe et al. (2004) for details on the data reduction. The individual spectra were also used to derive both the Bisector Inverse Slope (BIS) of the HARPS Cross-Correlation Function (CCF), as defined by Queloz et al. (2001), as well as a measurement of the chromospheric activity index $\log R_{\mathrm{HK}}^{\prime}$, following the same recipe used by Santos et al. (2000) for CORALIE spectra. Finally, the combined high S/N HARPS spectra were analyzed to derive stellar atmospheric parameters and iron abundances using the method described in Santos et al. (2004b).

\section{A new planet around HD 219828}

\subsection{Stellar characteristics}

According to the Hipparcos catalog (ESA 1997), HD 219828 is a G0IV star with a parallax $\pi=12.33 \pm 1.01$ mas, an apparent magnitude $m_{v}=8.04$, and a colour index $B-V=0.654$. From these parameters, and taking the bolometric correction from Flower (1996), we derive an absolute magnitude $M_{v}=3.49$ and a luminosity of $3.34 L_{\odot}$.

The analysis of our combined HARPS spectra, with a total $\mathrm{S} / \mathrm{N}$ ratio above 500 , provide $T_{\text {eff }}=5891 \pm 18, \log g=4.19 \pm$ 0.02 , and $[\mathrm{Fe} / \mathrm{H}]=0.19 \pm 0.02$. Along with the above mentioned absolute magnitude, the Geneva Evolutionary models (Schaerer et al. 1993) indicate that HD 219828 has a mass of $1.24 M_{\odot}$ and an age of $5.2 \mathrm{Gyr}$. In Table 1 we summarize the stellar parameters derived for this star.

HD 219828 shows a slightly lower spectroscopic gravity as compared to main-sequence dwarf stars of the same temperature. An even lower gravity $(\log g=4.03)$ is obtained if the isochrone
Table 1. Stellar parameters for HD 219828.

\begin{tabular}{lcc}
\hline \hline Parameter & Value & Reference \\
\hline Spectral type & G0IV & Hipparcos \\
Parallax [mas] & $12.33 \pm 1.01$ & Hipparcos \\
Distance [pc] & 81.1 & Hipparcos \\
$m_{v}$ & 8.04 & Hipparcos \\
$B-V$ & 0.654 & Hipparcos \\
$M_{v}$ & 3.49 & - \\
Luminosity $\left[L_{\odot}\right]$ & $3.34 \dagger$ & - \\
$\operatorname{Mass}\left[M_{\odot}\right]$ & 1.24 & Schaerer et al. $(1993)$ \\
$\log R_{\mathrm{HK}}^{\prime}$ & -5.04 & HARPS \\
$v \sin i\left[\mathrm{~km} \mathrm{~s}^{-1}\right]$ & $2.9 \dagger \dagger$ & - \\
$T_{\mathrm{eff}}[\mathrm{K}]$ & $5891 \pm 18$ & - \\
$\log g$ & $4.18 \pm 0.02$ & - \\
$\xi_{\mathrm{t}}$ & $1.18 \pm 0.02$ & - \\
{$[\mathrm{Fe} / \mathrm{H}]$} & $+0.19 \pm 0.03$ & - \\
\hline
\end{tabular}

$\dagger$ Using the bolometric correction of Flower (1996).

$\dagger \dagger$ From HARPS spectra using a calibration similar to the one presented by Santos et al. (2002).

mass is used in conjunction with the stellar parallax (e.g. Santos et al. 2004b). This result is compatible with the spectral classification of the star (G0IV - ESA 1997), and indicates that HD 219828 may be slightly evolved. The stellar radius estimated using the luminosity-temperature-radius relation is $1.76 R_{\odot}$.

From the HARPS spectra we derived both a chromospheric activity index $\left(\log R_{\mathrm{HK}}^{\prime}=-5.04\right)$ and an estimate of the projected rotational velocity of the $\operatorname{star}\left(v \sin i=2.9 \mathrm{~km} \mathrm{~s}^{-1}\right)$. From the activity level and the $B-V$ colour we derive an age of $6.5 \mathrm{Gyr}$ (Henry et al. 1996) (at least above 2 Gyr - Pace \& Pasquini 2004), and a rotational period of 26 days (Noyes et al. 1984). All these values suggest that HD 219828 is an old chromospherically quiet star. According to the Hipparcos catalog, this star is considered to be constant in photometry, with a scatter of only 0.013 mag (typical for a constant star of its magnitude).

\subsection{HARPS orbital solution}

HD 219828 was observed 22 times with the HARPS spectrograph, between May 2005 to August 2006. Each measurement was done using an exposure time of $900 \mathrm{~s}$, in order to average out the stellar oscillation noise (e.g Bouchy et al. 2005). The complete radial velocity measurements obtained and the errors are presented in Table 2 . It is worth noticing that the errors quoted in Table 2, which are used to plot the error bars, refer solely to the instrumental (calibration) and photon-noise error share of the total error budget (e.g. activity and/or stellar oscillations are not considered, given the difficulty in having a clear estimate of their influence).

A quick analysis of the data revealed the presence of a stable 3.83-day period radial-velocity signal. This signal is best fitted using a Keplerian fit with an amplitude $K$ of $7.0 \mathrm{~m} \mathrm{~s}^{-1}$, and a non-significant eccentricity (see Fig. 1; leaving the eccentricity as a free parameter in the orbital fit provides a value of $0.11 \pm$ 0.09). This corresponds to the expected signal induced by the presence of a 19.8 Earth-mass (minimum-mass) companion to HD 219828 (Table 3).

Our radial velocity measurements as a function of Julian Date are shown in Fig. 2. The plot shows that, in addition to the short period signal, there is a long-period trend superimposed. We have tried to fit a two Keplerian model to the whole radial-velocity set using the stakanof genetic algorithm, recently developed by Tamuz et al. (in preparation; see also 
Table 2. Radial-velocity measurements of HD 219828 and HD 102195.

\begin{tabular}{ccc}
\hline \hline $\mathrm{JD}$ & $V_{\mathrm{r}}\left[\mathrm{km} \mathrm{s}^{-1}\right]$ & $\sigma\left(V_{\mathrm{r}}\right)\left[\mathrm{km} \mathrm{s}^{-1}\right]$ \\
\hline $\mathrm{HD} 219828$ & & \\
2453509.928056 & -24.03256 & 0.00066 \\
2453510.928367 & -24.01790 & 0.00091 \\
2453701.542843 & -24.02993 & 0.00041 \\
2453702.532514 & -24.01817 & 0.00046 \\
2453703.531084 & -24.01824 & 0.00036 \\
2453704.530683 & -24.02639 & 0.00040 \\
2453705.544423 & -24.02791 & 0.00035 \\
2453706.530181 & -24.01890 & 0.00045 \\
2453707.529860 & -24.02140 & 0.00059 \\
2453708.551863 & -24.03220 & 0.00046 \\
2453709.539910 & -24.02877 & 0.00057 \\
2453710.539622 & -24.01823 & 0.00049 \\
2453930.824911 & -24.05650 & 0.00066 \\
2453931.779782 & -24.05201 & 0.00104 \\
2453932.776323 & -24.04571 & 0.00065 \\
2453933.735140 & -24.04473 & 0.00080 \\
2453934.739643 & -24.05830 & 0.00070 \\
2453935.739712 & -24.05720 & 0.00069 \\
2453936.730288 & -24.04521 & 0.00076 \\
2453946.762931 & -24.06075 & 0.00071 \\
2453951.833961 & -24.05168 & 0.00074 \\
2453975.734459 & -24.05942 & 0.00069 \\
\hline HD 102195 & & \\
2453501.574413 & 2.15457 & 0.00037 \\
2453503.580640 & 2.06803 & 0.00133 \\
2453504.587954 & 2.15706 & 0.00082 \\
2453506.621511 & 2.08172 & 0.00146 \\
2453550.535485 & 2.17258 & 0.00075 \\
2453551.523110 & 2.11355 & 0.00117 \\
2453757.789800 & 2.06038 & 0.00038 \\
2453761.827482 & 2.05137 & 0.00036 \\
2453765.771759 & 2.07657 & 0.00037 \\
2453785.772065 & 2.13058 & 0.00037 \\
2453788.796126 & 2.16390 & 0.00033 \\
2453927.478651 & 2.08383 & 0.00062 \\
2453930.461429 & 2.05648 & 0.00062 \\
2453931.470554 & 2.06908 & 0.00067 \\
2453932.485026 & 2.17049 & 0.00065 \\
2453933.465358 & 2.18322 & 0.00069 \\
2453934.470134 & 2.08446 & 0.00062 \\
2453935.471600 & 2.06340 & 0.00065 \\
2453936.476701 & 2.13495 & 0.00061 \\
\hline & &
\end{tabular}

Pepe et al. 2007). Although the 3.83-day period signal was always present in the solutions, several similar quality solutions were found for the long period signal. These always ranged from $\sim 180$ to $\sim 800$ days in period, corresponding to the presence of a planet in the Jupiter-mass domain $\left(\sim 0.7 M_{\text {Jup }}\right)$. The best fit (with an rms of only $1.2 \mathrm{~m} \mathrm{~s}^{-1}$ ) gave a period of 181-days, eccentricity of 0.3 , and amplitude $K=21.6 \mathrm{~m} \mathrm{~s}^{-1}$ for the long period orbit.

Given the ambiguity in the result, we prefer at this point to fit a simple quadratic drift to the residuals of the short period signal. The global rms of the Keplerian + drift solution is $1.7 \mathrm{~m} \mathrm{~s}^{-1}$. We note, however, that this long term trend does not perfectly fit the observed variation; some of the two Keplerian models had better $\chi^{2}$ than the adopted preliminary solution. This is also illustrated by the increasing residuals of the fit as a function of time (Fig. 2). These facts explain the relatively high rms of the orbital solution found, and may imply that the orbital parameters (e.g. $e$ and $T$ ) of the short period planet may change slightly when the long period solution is better constrained.
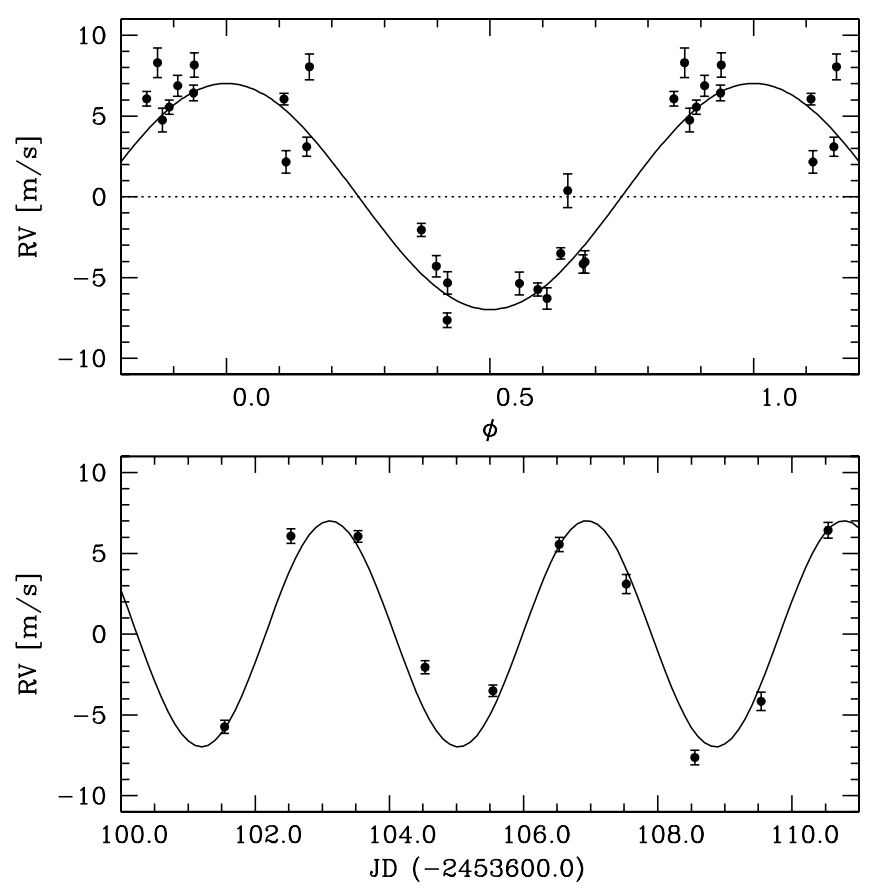

Fig. 1. Top: phase-folded radial-velocity measurements of HD219828, and the best Keplerian fit to the data with a period of 3.8-days. In this fit the long term radial-velocity trend was subtracted. See text for more details. Bottom: radial-velocity measurements of HD 219828 as a function of time for one epoch of measurements.

Table 3. Elements of the fitted orbit for HD 219828b.

\begin{tabular}{lll}
\hline \hline$P$ & $3.8335 \pm 0.0013$ & $\mathrm{~d}$ \\
$T$ & $2453898.6289 \pm 0.072$ & $\mathrm{~d}$ \\
$e$ & $0.0^{\dagger}$ & \\
$V_{\mathrm{r}}$ & $-24.032 \pm 0.001$ & $\mathrm{~km} \mathrm{~s}^{-1}$ \\
$\omega$ & $0.0^{\dagger}$ & $\mathrm{degr}$ \\
$K_{1}$ & $7.0 \pm 0.5$ & $\mathrm{~m} \mathrm{~s}^{-1}$ \\
$f_{1}(m)$ & $0.1364 \times 10^{-12} \pm 0.3572 \times 10^{-12}$ & $M_{\odot}$ \\
$\sigma(\mathrm{O}-\mathrm{C})$ & 1.7 & $\mathrm{~m} \mathrm{~s}^{-1}$ \\
$N$ & 22 & \\
$m_{2} \sin i$ & 19.8 & $M_{\oplus}$ \\
\hline
\end{tabular}

${ }^{\dagger}$ Fixed to zero. The obtained eccentricity was consistent with a circular orbit according to the Lucy \& Sweeney (1971) test.

To understand if the short period radial-velocity signal observed could be due to the presence of stellar spots (e.g. Saar \& Donahue 1997; Queloz et al. 2001) or stellar blends (Santos et al. 2002), we computed the Bisector Inverse Slope (BIS) of the HARPS Cross-Correlation Functions (CCF) of HD 219828. In Fig. 3 we plot the resulting BIS as a function of the radialvelocity, after having subtracted the long period quadratic trend to the data. The result shows that no correlation exists between the two quantities, suggesting that stellar activity of stellar blends cannot explain the short period and low amplitude radialvelocity variation observed.

Together with the very low activity level of the star, we thus conclude that the 3.83-day orbital period observed can be better explained by the presence of a Neptune-mass planet orbiting HD 219828. 


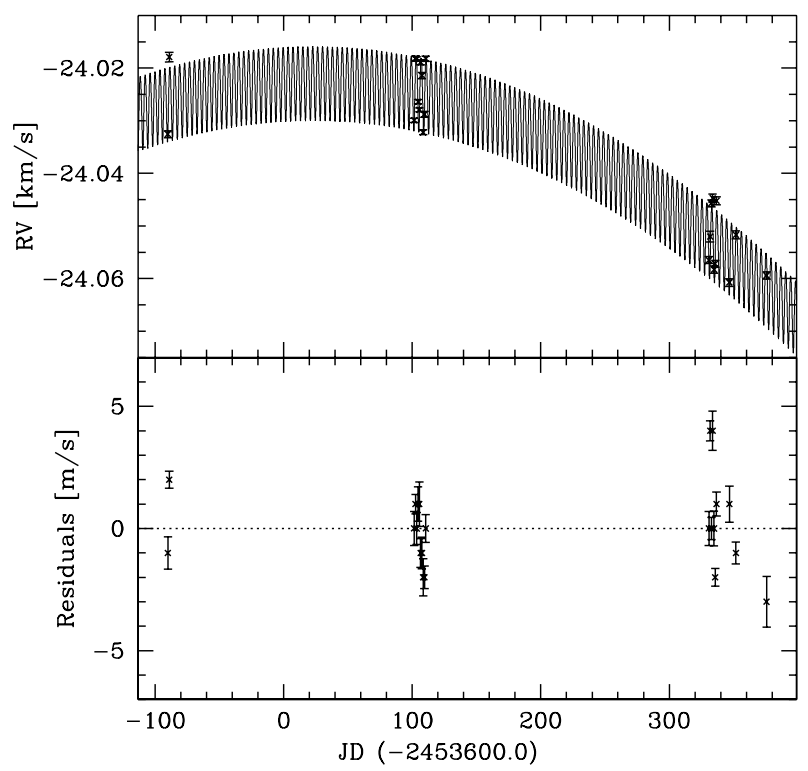

Fig. 2. Top: radial-velocity measurements of HD219828 as a function of time, and a fit to the data including a 3.8-day period Keplerian and a long period quadratic trend. Bottom: residuals of the fit.

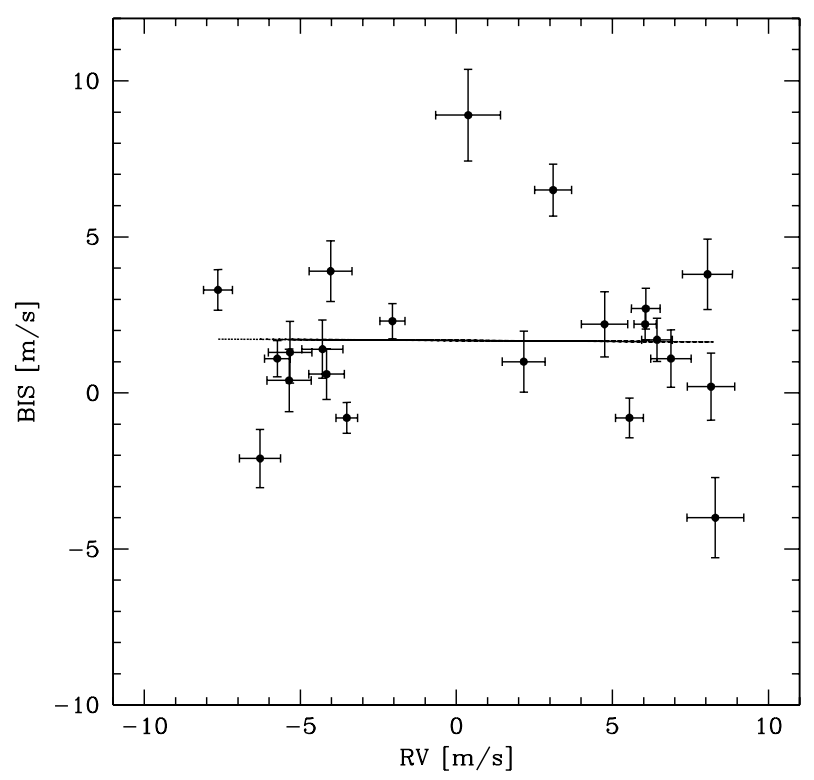

Fig. 3. BIS vs. radial-velocity for HD 219828. The best linear fit to the data is shown, and has a slope compatible with zero.

\section{The planet around HD 102195}

\subsection{Stellar characteristics of HD 102195}

According to the Hipparcos catalog (ESA 1997), HD 102195 is a K0V star with visual magnitude $m_{v}=8.07$ and colour index $B-$ $V=0.835$. From the visual magnitude and astrometric distance of $28.98 \mathrm{pc}(\pi=34.51 \pm 1.16)$, we derive an absolute magnitude $M_{v}=5.76$. Considering the bolometric correction taken from Flower (1996), the obtained luminosity is then $0.47 L_{\odot}$.

Accurate stellar atmospheric parameters for HD 102195 were derived from the analysis of our combined HARPS spectra, with a $\mathrm{S} / \mathrm{N}$ ratio above 300 . Our analysis provides $T_{\text {eff }}=$ $5291 \pm 34 \mathrm{~K}, \log g=4.45 \pm 0.04$, and $[\mathrm{Fe} / \mathrm{H}]=0.05 \pm 0.05$, in excellent agreement with previous estimates (Ge et al. 2006). This value for the gravity is compatible with the one derived
Table 4. Stellar parameters for HD 102195.

\begin{tabular}{lcc}
\hline \hline Parameter & Value & Reference \\
\hline Spectral type & K0V & Hipparcos \\
Parallax [mas] & $34.51 \pm 1.16$ & Hipparcos \\
Distance [pc] & 28.98 & Hipparcos \\
$m_{v}$ & 8.07 & Hipparcos \\
$B-V$ & 0.835 & Hipparcos \\
$M_{v}$ & 5.76 & - \\
Luminosity $\left[L_{\odot}\right]$ & $0.47 \dagger$ & - \\
$\operatorname{Mass}\left[M_{\odot}\right]$ & 0.87 & Schaller et al. (1992) \\
$\log R_{\mathrm{HK}}^{\prime}$ & -4.56 & HARPS \\
$v \sin i\left[\mathrm{~km} \mathrm{~s}^{-1}\right]$ & $2.6 \dagger \dagger$ & \\
$T_{\text {eff }}[\mathrm{K}]$ & $5291 \pm 34$ & \\
$\log g$ & $4.45 \pm 0.04$ & \\
$\xi_{\mathrm{t}}$ & $0.89 \pm 0.05$ & \\
{$[\mathrm{Fe} / \mathrm{H}]$} & $0.05 \pm 0.05$ & \\
\hline
\end{tabular}

$\dagger$ Using the bolometric correction of Flower (1996).

$\dagger \dagger$ From HARPS spectra using a calibration similar to the one presented by Santos et al. (2002).

using the temperature, astrometric distance, and stellar luminosity $(\log g=4.56)$. A stellar mass of $0.87 M_{\odot}$ is derived from the absolute magnitude, effective temperature, and metallicity, after comparison with stellar evolution models (Schaller et al. 1992). The stellar radius estimated using the luminosity-temperatureradius relation is $0.82 R_{\odot}$. According to the properties summarized in Table 4, HD 102195 is a typical solar metallicity mainsequence dwarf.

The HARPS cross-correlation function gives an estimate of the projected stellar rotational velocity $v \sin i=2.6 \mathrm{~km} \mathrm{~s}^{-1}$, a value that is reasonably compatible with the one derived by $(\mathrm{Ge}$ et al. 2006). Using HARPS spectra, we further derive a rather high value for the chromospheric activity index for this star $\left(\log R_{\mathrm{HK}}^{\prime}=-4.56\right)$. A similarly high value of $\log R_{\mathrm{HK}}^{\prime}=-4.30$ was obtained by Strassmeier et al. (2000).

Using the derived chromospheric activity index, and the colour of the star, we used the Henry et al. (1996) calibration to derive an estimate for the stellar age of $1.17 \mathrm{Gyr}$, and a rotational period of about 20-days (Noyes et al. 1984), slightly above the value found using the photometric light curve $(\sim 12$-days $-\mathrm{Ge}$ et al. 2006). As an additional age indicator, the Li abundance was computed as described in Israelian et al. (2004). We found a Li abundance $\log \epsilon(\mathrm{Li})<0.2$. According to Sestito \& Randich (2005), a $T_{\text {eff }} \sim 5300 \mathrm{~K}$ star with its lithium content should have an age above $0.5 \mathrm{Gyr}$, also compatible with the estimate of $\mathrm{Ge}$ et al. (2006).

\subsection{The HARPS orbital solution}

The discovery of a giant planet around HD 102195 has been recently announced by the Exoplanet Tracker team ${ }^{1}$ using a new concept of instrument combining spectroscopy and interferometry (Ge et al. 2006). Since this star is among our surveyed sample, it is interesting to compare the results obtained by Ge et al. (2006) with those obtained with HARPS.

We observed HD 102195 four times in May 2005, in the framework of our program aimed at finding earth-mass planets with HARPS around a metallicity biased sample of stars. Based on these first four measurements, HD 102195 was clearly detected to be a radial velocity variable. These first four measurements did not show any correlation between the bisector and

${ }^{1}$ http://www.astro.ufl.edu/et/ 
Table 5. Elements of the fitted orbit for HD 102195b, using the original and the corrected radial-velocity measurements. See text for more details.

\begin{tabular}{llll}
\hline \hline & Original velocities & Corrected velocities & \\
\hline$P$ & $4.114225 \pm 0.000855$ & $4.113775 \pm 0.000557$ & $\mathrm{~d}$ \\
$T$ & $2453896.00 \pm 0.04$ & $2453895.96 \pm 0.03$ & $\mathrm{~d}$ \\
$e$ & $0.0^{\dagger}$ & $0.0 \dagger$ & \\
$V_{\mathrm{r}}$ & $2.113 \pm 0.002$ & $2.132 \pm 0.001$ & $\mathrm{~km} \mathrm{~s}^{-1}$ \\
$\omega$ & $0.0^{\dagger}$ & $0.0 \dagger$ & $\mathrm{degr}$ \\
$K_{1}$ & $64 \pm 3$ & $63 \pm 2$ & $\mathrm{~m} \mathrm{~s}^{-1}$ \\
$f_{1}(m)$ & $1.135 \times 10^{-10} \pm 0.169 \times 10^{-10}$ & $1.070 \times 10^{-10} \pm 0.103 \times 10^{-10}$ & $M_{\odot}$ \\
$\sigma(\mathrm{O}-\mathrm{C})$ & 9.4 & 6.1 & $\mathrm{~m} \mathrm{~s}^{-1}$ \\
$N$ & 19 & 19 & \\
$m_{2} \sin i$ & 0.46 & 0.45 & $M_{\text {Jup }}$ \\
\hline
\end{tabular}

$\dagger$ Fixed to zero. The obtained eccentricity was consistent with a circular orbit according to the Lucy \& Sweeney (1971) test.
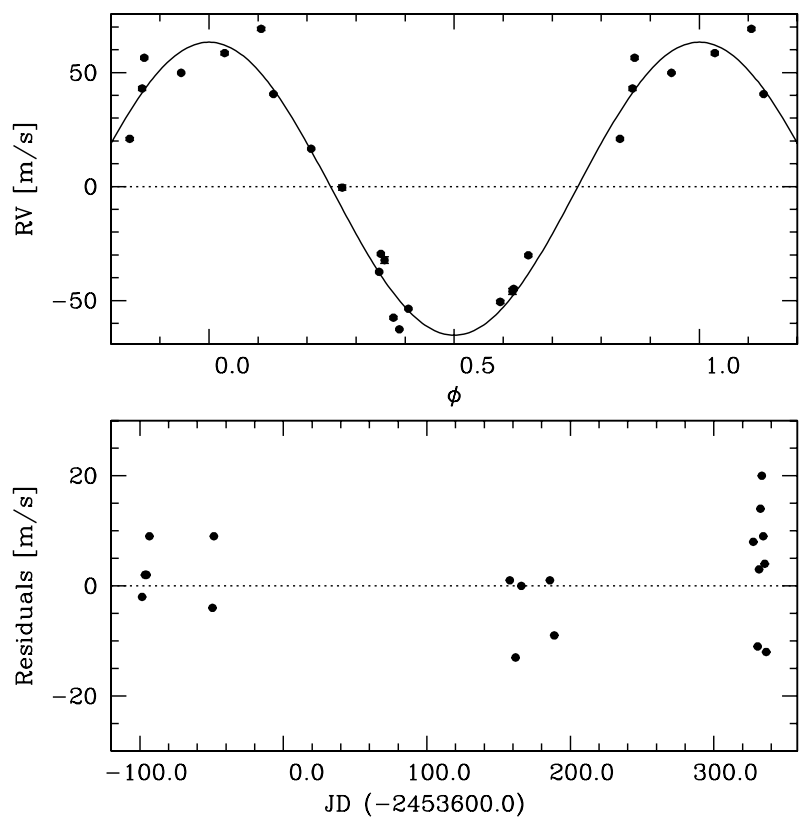

Fig. 4. Top: phase-folded radial-velocity measurements of HD 102195 , and the best Keplerian fit to the data with a period of 4.1-days. Bottom: residuals of the Keplerian fit as a function of time.

the radial velocities, suggesting that this star could be indeed a planet host.

The HARPS GTO team was contacted and kindly accepted to monitor HD 102195. In total, and counting also the data points obtained in our subsequent observing runs, 19 radial-velocity points were added between June 2005 and February 2006 in order to derive a good orbital solution. In the meanwhile, the star was announced as a planet host by Ge et al. (2006). In Table 2 we show the radial velocity measurements collected for HD 102195 between May 2005 and February 2006 covering a time span of about 300 days.

Using the radial velocity data shown in Table 2 an orbital solution was found (Table 5). The best fit Keplerian orbit gives a period of 4.11-days, an amplitude $\mathrm{K}$ of $64 \mathrm{~m} \mathrm{~s}^{-1}$, and a nonsignificant eccentricity. These values are similar to the ones derived by $\mathrm{Ge}$ et al. (2006). The phase-folded radial velocity plot of the measurements is shown in Fig. 4.

Despite the high activity level of the star, an analysis of the bisector of the HARPS cross-correlation functions shows no clear correlation with the radial-velocities (Fig. 5). This strongly suggests that the radial-velocity signal is not being produced

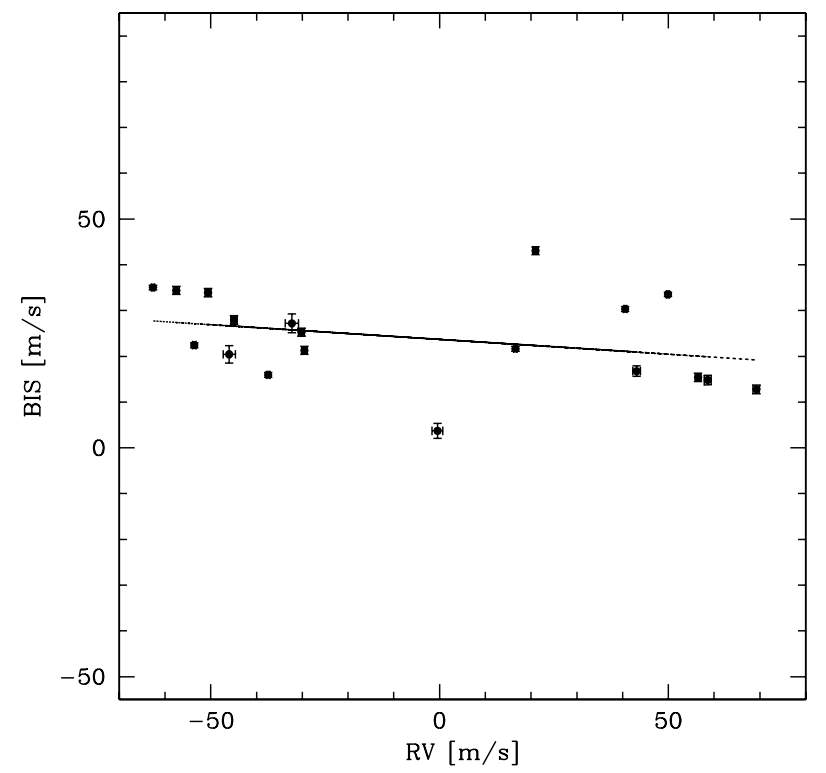

Fig. 5. BIS vs. radial-velocity for HD 102195 . The best linear fit to the data is shown, and has a slope compatible with zero.

by stellar spots or stellar blends, and supports the planetary explanation.

Using the best-fit orbital solution and the mass of HD 102195, we derive for the companion a minimum mass $0.46 M_{\mathrm{Jup}}$, in close agreement with the $0.49 M_{\mathrm{Jup}}$ found by Ge et al. (2006).

The stellar radius together with the rotational period mentioned above provide a value for the stellar rotational velocity of $\sim 3.5 \mathrm{~km} \mathrm{~s}^{-1}$. Comparing this with the estimated value for the projected rotational velocity $\left(v \sin i=2.6 \mathrm{~km} \mathrm{~s}^{-1}\right)$ we get $\sin i=0.74$, corresponding to an inclination of 47 degrees. If the orbital axis of the planetary orbit is aligned with the stellar rotation axis (e.g. Queloz et al. 2000), such an inclination could explain why no transit of HD $102195 \mathrm{~b}$ was detected by Ge et al. (2006). The real mass of planet would then be $0.62 M_{\text {Jup }}$.

\subsection{Correcting the radial velocities for the stellar noise}

In the case of HD 102195, the residuals $(\mathrm{O}-\mathrm{C})$ given in Table 5 of $9.4 \mathrm{~m} \mathrm{~s}^{-1}$ are much higher than the errors on radial velocity quoted in Table 2. This extra-noise is likely due to stellar activity (see previous section). Ge et al. (2006) found that this star has a photometric variation with a period of $\sim 12$ days, and an amplitude of $\sim 0.015 \mathrm{mag}$. 


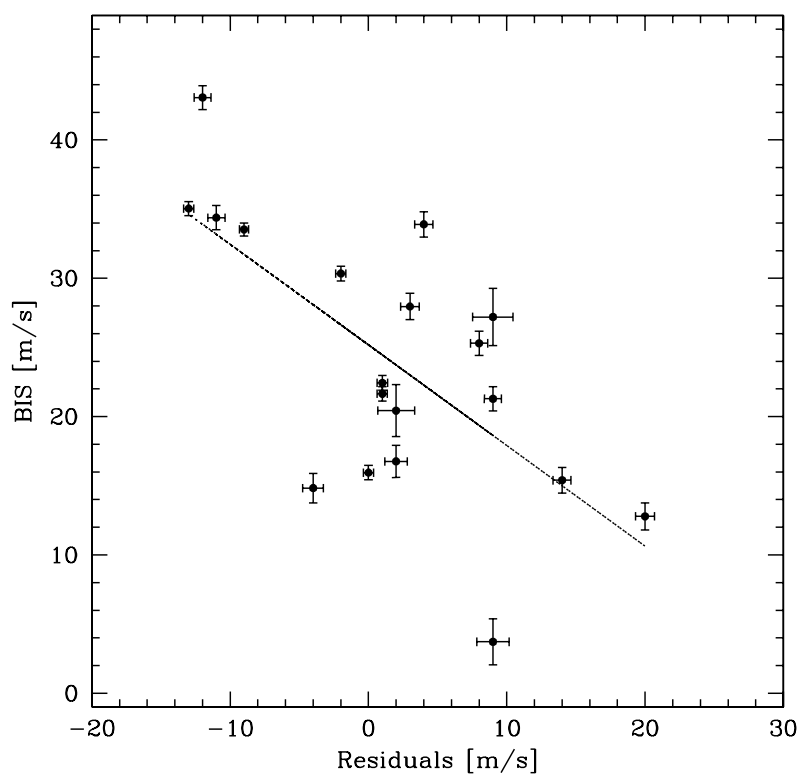

Fig. 6. BIS as a function of the residuals of the 4.1-day Keplerian radialvelocity fit for HD 102195. The data is clearly correlated. The best linear fit has a slope of -0.73 .

The signature of its high activity level is also seen on the bisector analysis done in this paper. Figure 6 shows that the residuals of the best Keplerian orbit (previous section) do correlate with the bisector measurements in a clear indication that the high-activity level of this star is responsible for the observed high residuals (e.g. Saar \& Donahue 1997; Santos et al. 2000).

According to Fig. 6, the relation between the residual radialvelocities and the BIS measurements can be approximated by a linear function $a \times B I S+b$ whose coefficients were determined by a least-squares fit. This function can be subtracted from the radial velocity data points in order to correct for the stellar activity.

Using those corrected velocities, a new orbital solution was found. The results, also presented in Table 5, show a clear improvement. Not only the rms around the orbital fit was reduced from 9.4 to $6.1 \mathrm{~m} \mathrm{~s}^{-1}$ (equivalent to subtracting quadratically a noise of $7 \mathrm{~m} \mathrm{~s}^{-1}$ ), but also the estimated errors in all orbital parameters decreased by almost a factor of two. The resulting mass for HD $102195 \mathrm{~b}$ also slightly decreased $\left(0.45 M_{\mathrm{Jup}}\right)$ due to the small decrease in the amplitude of the orbital solution.

This result shows that the study of the BIS can be used to correct, at least to some extent, the noise on the radial-velocities introduced by intrinsic stellar activity. If the BIS vs. RV correlation can be calibrated (observationally or theoretically) as a function of the stellar properties, we could think that in the future an externally determined BIS vs. RV function can be used to correct the radial velocity data. This will be extremely important in reaching accuracy of the other of the $\mathrm{cm} \mathrm{s}^{-1}$ as expected in future instruments like CODEX (Pasquini et al. 2005).

\section{Concluding remarks}

In this paper, we present the discovery of a new $20 M_{\oplus}$ (minimum-mass) short period planet, orbiting the sun-like star HD 219828. The presence of a second longer period companion to the system, likely a Jovian planet, is also clear from our data. Unfortunately, the bad phase coverage of the radialvelocity points does not permit to settle the orbital parameters of this latter companion. Finally, we confirm the existence

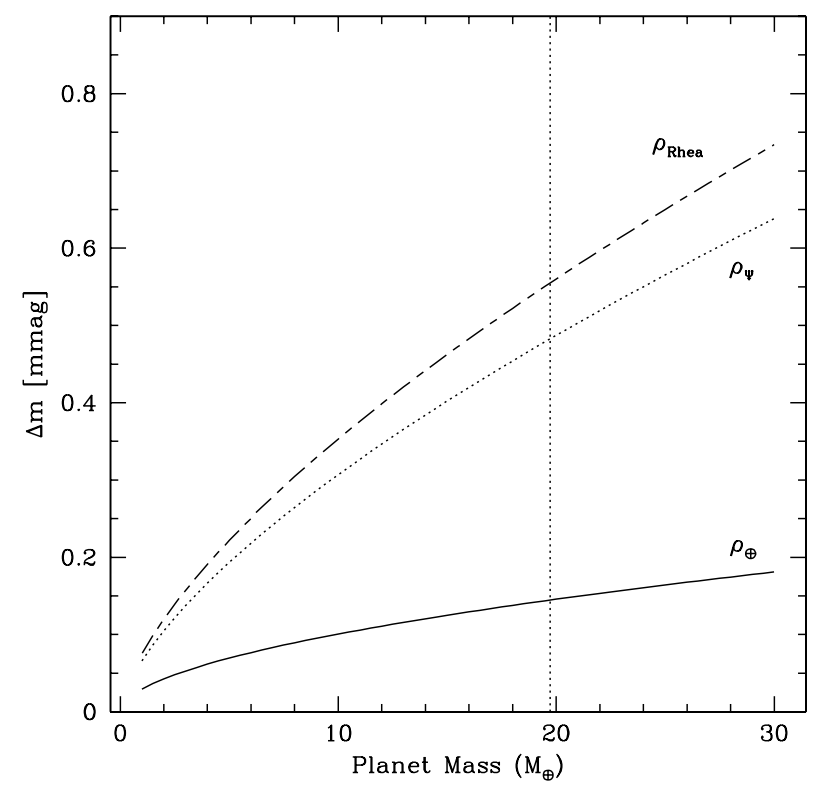

Fig. 7. Transit depth expected for three different planet models as a function of the mass. The lower curve represents the model of Valencia et al. (2006), and the two upper curves the scaled radii considering the mean density of Neptune and the icy Saturn satellite Rhea. The vertical dotted line represents the location of HD $219828 \mathrm{~b}$.

of a $m_{2} \sin i=0.45 M_{\text {Jup }}$ planet orbiting HD 102195, previously announced by another team.

The planet around HD 219828 is the 11th found with masses similar to the mass of Neptune. As the number of these systems increases, new statistical analysis will be possible. In this sense, Udry et al. (2006) have discussed the possibility that the well known strong correlation between the presence of planets and the stellar metallicity that exists for stars hosting giant planets (e.g. Gonzalez 1997; Santos et al. 2001, 2004b; Fischer \& Valenti 2005) does not seem to be present for their lower mass counterparts. For very-low mass companions, the metallicity distribution could rather be flat, something that may be explained by recent models of planet formation (Ida \& Lin 2004; Benz et al. 2006). Although the metallicity of HD 219828 is rather high, we must be careful when analyzing the numbers, since the planetsearch project described in this paper concentrated its efforts in studying a sample of metal-rich stars only.

An important question triggered by the previous discoveries is whether these small planets are constituted by rocks, ice or a mixture of both. A more solid assessment of the nature of HD 219828 b can only be given if the information about its mass and radius is available. The detection of a transiting signature would give us information about the radius and mean density of the planet, thus opening the possibility to unveil its composition, like is the case for the giant planets found to transit their host stars (e.g. Konacki et al. 2003; Pont et al. 2004).

Although the short period of the orbit implies a reasonable value for the transit probability $(\sim 15 \%)$, the measure of a transit of HD $219828 \mathrm{~b}$ is not an easy task. Using the models presented by Valencia et al. (2006), considering that this $m_{2} \sin i=$ $19.8 M_{\oplus}$ planet could have an Earth-like composition, we derive its radius to be $\sim 2.2$ Earth radii. Although this value must be taken as a lower limit (both due to the lower limit of the mass, and to the fact that it may have an extended atmosphere - Lovis et al. 2006), observing the transit of such a low-mass planet is likely impossible with current ground-based instrumentation (see Fig. 7). 
For the case of HD 102195, we have also tested, for the first time, the use of the bisector for the cross-correlation function to correct the radial-velocity measurements for the noise introduced by stellar activity. The results show that a considerable improvement can be found. Although the technique may probably be improved, this conclusion constitutes a first step towards the subtraction of the radial-velocity noise produced by stellar activity phenomena.

Acknowledgements. We thank the referee Dr. Barbara McArthur whose comments helped to improve our paper. Support from Fundação para a Ciência e a Tecnologia (Portugal) to N.C.S. and S.G.S. in the form of fellowships (references SFRH/BPD/8116/2002 and SFRH/BD/17952/2004) and grant (reference POCI/CTE-AST/56453/2004) is gratefully acknowledged. WG, GP and MTR were supported by the Chilean FONDAP Center of Astrophysics 15010003.

\section{References}

Alibert, Y., Baraffe, I., Benz, W., et al. 2006, A\&A, 455, L25

Benz, W., Mordasini, C., Alibert, Y., \& Naef, D. 2006, in Tenth Anniversary of 51 Peg-b: Status of and prospects for hot Jupiter studies, ed. L. Arnold, F. Bouchy, \& C. Moutou, 24

Bonfils, X., Udry, S., Delfosse, X., Santos, N., \& Mayor, M. 2005, A\&A, 443, L15

Bouchy, F., Bazot, M., Santos, N. C., Vauclair, S., \& Sosnowska, D. 2005, A\&A, 440, 609

Butler, R. P., Vogt, S. S., Marcy, G. W., et al. 2004, ApJ, 617, 580

ESA. 1997, The Hipparcos and Tycho Catalogues

Fischer, D. A., \& Valenti, J. 2005, ApJ, 622, 1102

Flower, P. J. 1996, ApJ, 469, 355

Ge, J., van Eyken, J., Mahadevan, S., et al. 2006, ApJ, 648, 683
Gonzalez, G. 1997, MNRAS, 285, 403

Henry, T. J., Soderblom, D. R., Donahue, R. A., \& Baliunas, S. L. 1996, AJ, 111, 439

Ida, S., \& Lin, D. N. C. 2004, ApJ, 616, 567

Israelian, G., Santos, N. C., Mayor, M., \& Rebolo, R. 2004, A\&A, 414, 601

Konacki, M., Torres, G., Jha, S., \& Sasselov, D. 2003, Nature, 421, 507

Lovis, C., Mayor, M., Pepe, F., et al. 2006, Nature, 441, 305

Lucy, L. B., \& Sweeney, M. A. 1971, AJ, 76, 544

McArthur, B. E., Endl, M., Cochran, W. D., et al. 2004, ApJ, 614, L81

Noyes, R. W., Hartmann, L. W., Baliunas, S. L., Duncan, D. K., \& Vaughan, A. H. 1984, ApJ, 279, 763

Pace, G., \& Pasquini, L. 2004, A\&A, 426, 1021

Pasquini, L., Cristiani, S., Dekker, H., et al. 2005, The Messenger, 122, 10

Pepe, F., Mayor, M., Queloz, D., et al. 2004, A\&A, 423, 385

Pepe, F., Correia, A. C. M., Mayor, M., et al. 2007, A\&A, 462, 769

Pont, F., Bouchy, F., Queloz, D., et al. 2004, A\&A, 426, L15

Queloz, D., Eggenberger, A., Mayor, M., et al. 2000, A\&A, 359, L13

Queloz, D., Henry, G. W., Sivan, J. P., et al. 2001, A\&A, 379, 279

Rivera, E. J., Lissauer, J. J., Butler, R. P., et al. 2005, ApJ, 634, 625

Saar, S. H., \& Donahue, R. A. 1997, ApJ, 485, 319

Santos, N. C., Mayor, M., Naef, D., et al. 2000, A\&A, 361, 265

Santos, N. C., Israelian, G., \& Mayor, M. 2001, A\&A, 373, 1019

Santos, N. C., Mayor, M., Naef, D., et al. 2002, A\&A, 392, 215

Santos, N. C., Bouchy, F., Mayor, M., et al. 2004a, A\&A, 426, L19

Santos, N. C., Israelian, G., \& Mayor, M. 2004b, A\&A, 415, 1153

Schaerer, D., Charbonnel, C., Meynet, G., Maeder, A., \& Schaller, G. 1993, A\&AS, 102, 339

Schaller, G., Schaerer, D., Meynet, G., \& Maeder, A. 1992, A\&AS, 96, 269

Sestito, P., \& Randich, S. 2005, A\&A, 442, 615

Strassmeier, K., Washuettl, A., Granzer, T., Scheck, M., \& Weber, M. 2000, A\&AS, 142, 275

Udry, S., Mayor, M., Benz, W., et al. 2006, A\&A, 447, 361

Valencia, D., O’Connell, R. J., \& Sasselov, D. 2006, Icarus, 181, 545

Vogt, S. S., Butler, R. P., Marcy, G. W., et al. 2005, ApJ, 632, 638 